\title{
The Anti-interference Study on D2D Communications
}

\author{
Xianjun Zhou, Ya Zhang, Qiulin Sheng, and Danwen Wu \\ School of Electrical \& Electronic Engineering, \\ Hubei University of Technology, Wuhan 430068, China; \\ Email: xianjun_zhou@sina.com
}

\begin{abstract}
D2D (Device-to-Device) communication is a new technology that allowing terminals communicate directly by reusing cell resources under the $B S$ (base station) controlling.After bringing D2D communication mode into cellular network, and that will cause interference problems. This paper describes the generation of interference after D2D communication mode being brought into the cellular network and then summarize current interference studies of a single cell and multi-cellular communications networks.
\end{abstract}

Keywords- Device-to-Device; anti-interference; single cell, multi-cellular;LTE (Long Term Evolution)

\section{INTRODUCTION}

The Ministry of Industry and Information Technology in China issued license TD-LTE to the three operators on December 4, 2013, which marks the arrival of $4 \mathrm{G}$ era in this country. Constantly evolving in mobile communication systems, along with the increasing demand for bandwidth, spectrum resources have become shortage. The spectrum resources are limited today, in order to further improve resources utilization, 3GPP standardization organization have launched a series of work, such as reconstruction of heterogeneous networks, cell clustering and so on. Operators prefer to the smooth evolution of the system rather than investing a lot of money to the laying of small base stations. D2D technology can upgrade directly from the existing network.

Today,5G technology has attracted the people's attention,D2D technology is considered as a key technology on IMT-2020 study together with large-scale MIMO,ultra-dense deployments, flexible duplex and so on.D2D technology enables data transmit directly between terminals

by reusing cell spectrum resources. There are many advantages of combining with D2D system and cellular system. Such as expanding the cell capacity, improving spectrum utiliztion, effectively reducing the BS loading, reducing the mobile terminal battery power, shortening the delay, etc.And it also can support the new small-scale point-to-point data services.

D2D communication and Bluetooth technology can transmit data directly in short distances,but Bluetooth uses unlicensed spectrum and requires the users to manual pairing to communicate.Compared with WLAN, WLAN needs to customized set a access point AP before communication and it also uses unlicensed spectrum.But D2D communication don't need the above process and it uses licensed spectrum.TheD2D communications' interference environment can be controlled and the data transmission is more reliable. In addition, compared with the cognitive radio, cognitive radio technology reuse the idle spectrum that main network is not occupied. But D2D cellular communication technology is shared spectrum resources. Therefore,D2D communication will become a key technology in the future mobile communications.

\section{THE GENERATION OF INTERFERENCE}

Bringing D2D communication into cellular network, D2D users can communicate directly by reusing cell resources. Then there are three communication modes for all the users on the cellular.Namely cellular communication mode, D2D non-orthogonal resource sharing model and D2D orthogonal resource sharing[1].

When D2D users reusing the cellular resources, if the $\mathrm{D} 2 \mathrm{D}$ user is assigned to a orthogonal spectrum resource, then it will not cause any interference to the original cellular network communications.But if the D2D user is assigned to a non-orthogonal spectrum resource, D2D communication will cause interference to the original cellular users. Therefore, if the load is small on the communication network, the network can achieve the better performance while allocated excess orthogonal resources for D2D communications. However, due to the limited spectrum resources on a cellular network, the frequency bandwidth on communication services have become increasingly demanded. So the non-orthogonal resource sharing model is widely used and it can make the network have higher resource utilization. Therefore, the solution of the interference generated by non-orthogonal resource sharing have become a key issue which is urgently needed to be addressed.

In D2D communication system, after BS allocate spectrum resources to $\mathrm{D} 2 \mathrm{D}$ users,

D2D users can choose to reuse uplink spectrum resources or downlink spectrum resources of the cellular network. 
The interference between D2D users and the cellular users is different which based on the reusing uplink spectrum resources or downlink spectrum resources. When reusing uplink spectrum resource, the cellular uplink users will have an interference on D2D receivers and so as the D2D transmitters to the BS, as shown in Fig .1. When reusing downlink spectrum resource, BS will have an interference on D2D receivers and so as the D2D transmitters to the cellular downlink users, as shown in Fig .2[2,3]

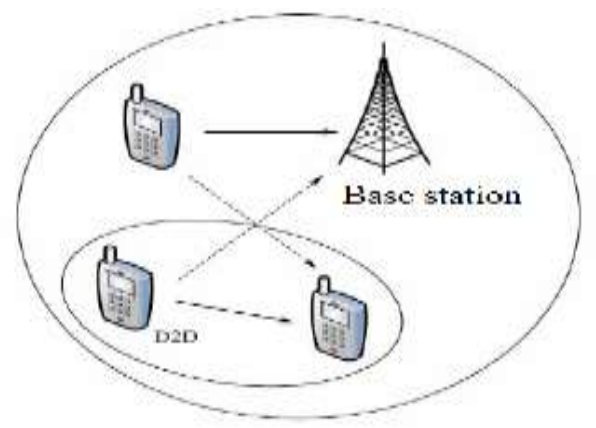

Figure 1.The schematic diagram of interference when D2D reusing uplink spectrum resources

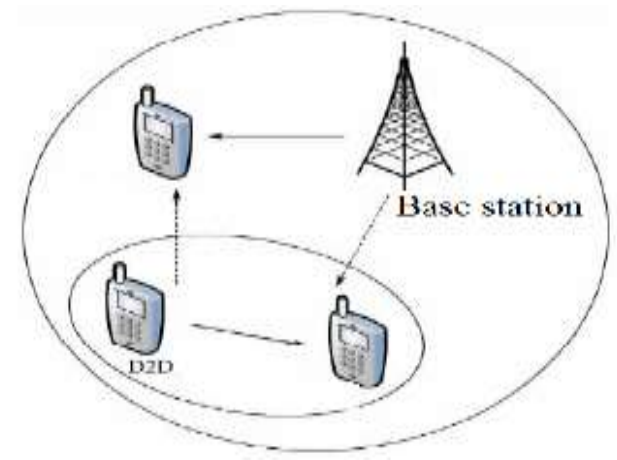

Figure 2.The schematic diagram of interference when D2D reusing downlink spectrum resources

D2D communication users based on reusing different cellular uplink or downlink spectrum resources have advantages and disadvantages.

For example, the location of the D2D users in cellular network have little influence on D2D users' transmission power when D2D users reusing downlink resource. But it's much more sensitive to reuse uplink resources than downlink resources. When the communication users are far away from the BS, the curve of gain ratio between D2D communication user's SINR signal and interference-plus-noise with D2D reusing uplink resources better than reusing downlink.On the contrary, when the communicat-

ion users are closer to the BS, reusing downlink resources better than reusing uplink. Therefore, we can allow users to reuse downlink spectrum resources when D2D communication users are close to the BS, but reuse uplink spectrum resources when D2D communication users are far away from the BS.

\section{SINGLE-CELL CELLULAR NETWORK ANTI-INTERFERENCE STUDIES}

When the resources of cellular users and D2D users are non-orthogonal, it will cause interference each other. In order to ensure normal cellular users' communications, we usually set a minimum rate threshold to the cellular users. Otherwise it may lead to the interrupt to cellular communications[4]. Generally, we solve the interference mainly through power control and resource allocation. Also there are some other techniques, such as: multi-antenna technology, advanced coding technology and so on. Single-cell cellular network shown in Fig .3.

In this model, it contains a BS, three RN (Relay Node) , a tradition cellular user UE1 and one or more pairs of D2D users UE2- UE3. While RN complete the relay function between the temporary mobile BS and the fixed BS. Based on consideration of enhancing the coverage of original cell. RN is usually placed near the cell edge, and only provide a relay service to conventional cellular users.

In [5],in order to avoid or reduce the interference, the author proposed an optimal model selection strategy by establishing a "Dynamic Mapping Users" in the local database with BS, which used to promptly keep track of the situations of all cellular band be reused and then D2D device actively choose to reuse the cellular resources that have a low level interference. In this mechanism and algorithm design, cellular users QoS is full considered, as well as the mutual interference between the cellular and D2D uses.In [6], using the users' space isolation in the cellular network, in order to reduce the interference between two modes of users' communication, starting from the perspective of resource scheduling, picking out cellular users and D2D communication users to reuse resources reasonably. we propose a interference suppression that suit D2D communication reusing downlink resources on cellular network. This method takes advantage of the user's spatial isolation to reduce interference between two modes of communicat-

ion and improves overall cell throughput.In [7] proposed a solution to solve the interference problem between D2D user groups. Firstly, we divide these D2Dusers into different user groups based on location of D2D users on the edge the cellular. Secondly, allocate orthogonal or non-orthogonal resources to different D2D groups of users. Finally, applying different resource allocation algorithms based on the different resources that allocated to D2D user in the groups. In[6] a D2D communication model mapping scheme is proposed. With this scheme, the BS can quickly decide reasonable communication model based on the user's location in the communication system, and assign appropriate resources. In this model mapping scheme, it will loss some limited performance gain but it can greatly reduce complexity of the mode decision .In [7], based on multi-antenna of single-cell cellular network scenario, which proposed two types of precoding algorithm with D2D users reusing downlink spectrum resources to eliminate the 
interference. One is exhaustive searching all known precoding codebook, using the best pre-coding. This scheme has a overhead to the system. Another is adopted a distributed algorithm, if we can get accurate channel feedback,compared to exhaustive searching for the optimal pairs of pre-coded, it will get a higher gain.In [8], under LTE-Advanced network with Underlay D2D communication scenario. When D2D communic-

ations reuse uplink resources,BS adjust the transmission power of D2D communications and the cellular communications to control interference in which case the BS controls transmission power of D2D communications reduced a threshold to ensure that the SINR on uplink is greater than the target SINR in system. When D2D communications reuse downlink resources, BS pre-sets a value of $\mathrm{D} 2 \mathrm{D}$ maximum transmission power, this values of the maximum transmission power is larger than values of transmission power when the D2D users reuse the a idle spectrum cellular resources. When allocat the resources, the BS will separate D2D users and cellular users in propagation space.

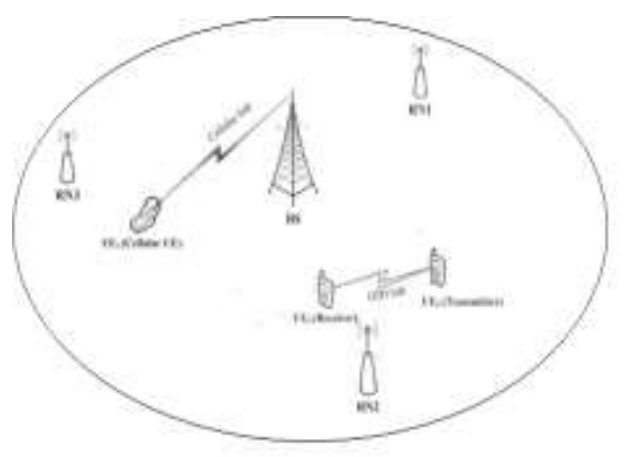

Figure 3.the mode of single-cell cellular network

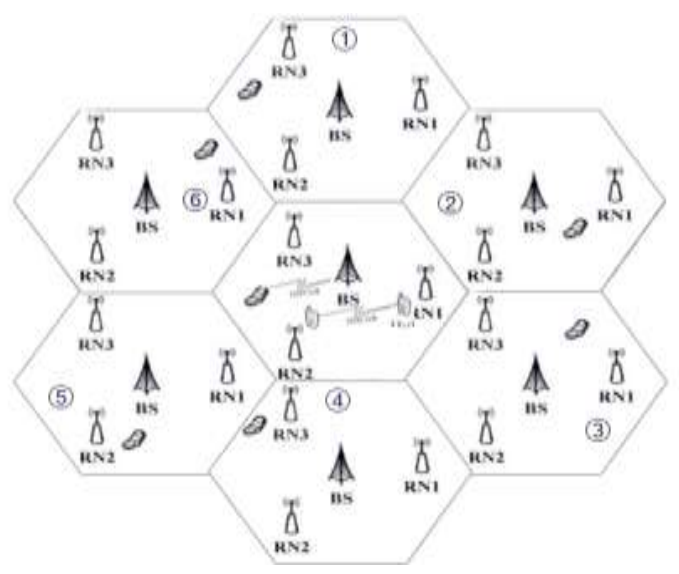

Figure 4.the mode of multi-cell cellular network

\section{MULTI-CELL CELLUAR NETWORK ANTI-INTERFERENCE STUDIES}

As single-cell cellular network model is too ideal and single, multi-cell cellular network model is more realistic. Multi-cell cellular network interference studies described as below. multi-cell cellular network node as shown in Fig .4, In this model,a hexagonal is used to describe the coverage area of cell. The interference in any cell mainly come from those nearest cell, i.e.six surrounding adjacent cells

An interference coordination mechanism and a mode selection mechanism between D2D users and cellular users under the help of local network are proposed in [5]. Non-local cell target cellular users sent applications for assistance to the local cellular BS by the BS.The local BS and the target BS exchange D2D communication information,then feedback the allocation resources to the D2D communications. This mechanism effectively ensure the quality of traditional cellular service users ,even more fully take advantage of all available resources in the multi-cell networks, and greatly enhance the throughput and spectral utilization of hybrid networks. In [11] proposed a mode selection mechanism that through comparing the throughput of various modes which determined by the base station in multi-cell environment. thus ensured a reliable D2D communication and limited interference of cellular networks.[12] proposed a communicati-

on mode selection algorithm based on the location of D2D users in single cellular. With the basis of his mode selection algorithm proposed a resource allocation algorithm and power control algorithm which used of the loss among users as the only reference factor in multi-cell cellular communication network. Combining to this two algorithm can coordinate inter-cell communication interference.This method can greatly improve the system performance and well coordinate the interference in the system.

\section{SUMMARY}

After bringing D2D communication technology into the traditional cellular communication system, as D2D communication technology reuse cellular communication resources which will cause interference problems in the cell. In this paper we analyze the generation of interference after the bringing D2D communication into the cellular network. And then summarize the anti-interference study on D2D communication.As applied D2D communication technology into the cellular communication system, the throughput of the system will be effectively enhanced.We can improve spectrum utilization by reusing spectrum resources.More importantly, realization of D2D communication in cellular network can provide more extensive interconnected network in the future and may cause generation of new communication business.

\section{REFERENCES}

[1]Xu Y .,Yin R., Han T. \& Y u G .Interference-aware channel allocation for device-to-device communication underlaying cellular networks[C]. IEEE International Conference on Communications in China (ICCC), 2012:422-427. 
[2]Wang Junyi, Gong Zhishuai, Fu Jenin, Chen Xiaohui, Lin Jiming. The Survey of D2D communication [J]. Guilin University of Electronic Technology, 2014, 4 (2) : 114-119

[3]Jiao Yan, GaoYuehong, Yang Hongwen, Yang Dacheng.D2D technology research status and development prospects [J]. Technology and Business Innovation, 2014, 4(6): 83-87

[4] $\mathrm{Xu}$ Tonggang. The interference study after D2D technology introduced into the LTE system[J] . Telecom Express, 2012(11): 32-35

[5]Xiang Shangwen.Mode selection and interference coordination in the hybird D2D and cellular network [D] master's thesis, Beijing University of Posts and Telecommunications, 2012

[6]Nie Gaofeng,Lu Bo,Yang Guang.A D2D communication anti-interference method of reusing downlink resource in cellular network[J],Telecommunication Network Technology, 2012, 8(8): 54-59

[7]Zhu XiaoyuePerformance Research of D2D in Cellular Networks[D].Master Thesis, Beijing University of Posts and Telecommunications, 2013, 3
[8]Yuan chenjun,Wang Xiaoxiang,Wang Dongyu. Resource Allocation Scheme Based on User Grouping for Device -to -Device Communica-tion[J]. Journal of Chengdu University of Inform Ation Technology, 2012.12(27):594-598

[9]The study of interference suppressio technique of communication underlaying cellular network[D]. Master Thesis.University of Electronic Science and Technology, 2014, 6

[10]Klaus Doppler, Mika Rinne, Carl Wijting, CássioB.Ribeiro,and Klaus Hugl.Device-to- Device communication as an underlay to LTE- Advanced networks[J].IEEE Communications Magazine.December2009:42-49

[11]Doppler K,Yu C H, Ribeiro C B,etal. Mode selection for Device-to-Device communication underlaying an LTE-advanced Network[C]. Wireless communications and Networking Conference (WCNC), 2010:1-6.

[12]Tu Tianming. Suitable for D2D communication multiplexing method with interference suppression cellular network by reusing downlink resources [J]. New Technology. 2013.02 